\title{
Frucht Graph is not Hyperenergetic
}

\author{
S. PIRZADA \\ Department of Mathematics, University of Kashmir, Kashmir, India \\ Email:sdpirzada@yahoo.co.in
}

\begin{abstract}
If $\lambda_{1}, \lambda_{2}, \ldots, \lambda_{p}$ are the eigen values of a p-vertex graph $G$, the energy of $G$ is $E(G)=\sum_{i=1}^{p} \lambda_{i}$. If $E(G)>2 p-2$, then $G$ is said to be hyperenergetic. We show that the Frucht graph, the graph used in the proof of well known Frucht's theorem, is not hyperenergetic. Thus showing that every abstract group is isomorphic to the automorphism group of some non-hyperenergetic graph. AMS Mathematics Subject Classification: $05 \mathrm{C} 50,05 \mathrm{C} 35$
\end{abstract}

Keywords: energy of a graph, hyperenergetic, frucht graph, automorphism

\section{Introduction}

The concept of hyperenergetic graphs was introduced by Gutman [1]. The existence of hyperenergetic graphs has been known for quite some time, their systematic design was first achieved by Walikar et al. [2]. Details can also be seen in [3-6].

The following result can be found in [4].

Theorem 1. A graph with $\mathrm{p}$ vertices and $\mathrm{m}$ edges such that $m<2 p-2$ is not hyperenergetic.

In this paper, we give the existence of one more class of hyperenergetic graphs, called the Frucht graphs.

\section{Frucht Graph is not Hyperenergetic}

Let $\Gamma$ be a group with $n$ elements and $\Lambda$ be a set of $\Gamma$ not containing the identity e. The Cayley digraph is defined to be the digraph with vertex set $V=\Gamma$ and arc set $A=\{(g, g h): h \in \Lambda\}$. It is denoted by $D=D(\Gamma, \Lambda)$. If $\Lambda$ $=\Gamma-\mathrm{e}$, the resulting Cayley digraph is complete and is denoted by $K=K(\Gamma, \Lambda)$. If $\Lambda$ is a set of generators for $\Gamma$, the Cayley digraph is called the basic Cayley digraph.

In the Cayley digraph $D=D(\Gamma, \Lambda)$, if $(g, h)$ is an arc, $k=g h$ for some $h \in \Lambda$, that is $g^{-1} k \in \Lambda$ and $g^{-1} k$ is called the color of $(g, k)$.

An automorphism of $D$ is said to be color preserving if it preserves the colors of the arcs. It is well known that the group $C(D)$ of color preserving automorphisms of the Cayley digraph $D=D(\Gamma, \Lambda)$ is isomorphic to $\Gamma$.

The following result is the Frucht's Theorem [7-9].

Theorem 2. Every group is isomorphic to the auto- morphism group of some graph.

While proving Theorem 2, Frucht obtained the graph $G_{1}$ from $D(\Gamma, \Lambda)$ called as Frucht graph, whose automorphism group is isomorphic to $C(D)$.

The following is the construction of Frucht graph $G_{1}$.

Replace each arc $g_{i} g_{j}$ of $D$ by a figure joining vertices $g_{i}$ and $g_{j}$. The figure consists of the 3-path $g_{i} u_{i} v_{i} g_{j}$, and two paths- a path $p_{2 k}$ (containing $2 k$ vertices) rooted at $u_{i}$, and a path $p_{2 k+1}$ (containing $2 k+1$ vertices) rooted at $v_{i}$, where $g_{i}^{-1} g_{j}=g_{k}$ is the color of $g_{i} g_{j}$. (Note that there will be a similar figure corresponding to $g_{j} g_{i}$ for a different $k$ ).

Clearly, the Frucht graph $G_{1}(\Gamma)$ with $\Lambda=s$ has $n(s+1)(2 s+1)$ vertices.

Theorem 3. The Frucht graph $G_{1}$ has $n s(2 s+1)$ edges.

Proof. The number of edges $m$ in $G_{1}(\Gamma)$ is given by

$$
m=\sum_{i=1}^{n} \sum_{k=1}^{s}(4 k-1)=n\left[\frac{4 s(s+1)}{2}-s\right]=n s(2 s+1)
$$

Theorem 4. The Frucht graph $G_{1}$ is not hyperenergetic.

Proof. We observe that,

$$
m=n s(2 s+1)<2[n(s+1)(2 s+)]-2=2 p-2 .
$$

Thus the result follows from Theorem 1 .

Lovasz [10] gives an alternate construction of the 
graph $G_{2}(\Gamma)$ used to prove the Fruchts theorem. In this case the figure of color $k$ is a path of length $k+2$, including the end vertices $g_{i}$ and $g_{j}$, in which to the first $k$ internal vertices are attached a path $P_{2}$ and to the last internal vertex (near $g_{j}$ ) is attached a path $P_{3}$.

Theorem 5. $G_{2}(\Gamma)$ has $n\left(s^{2}+4 s+1\right)$ vertices, where $|\Lambda|=s$.

Proof. In $G_{2}(\Gamma)$ each arc $g_{i} g_{j}$ is replaced by a figure with $k+1+2 k+3=3 k+4$ internal vertices(that is excluding $g_{i}$ and $g_{j}$ ) if $g_{i}^{-1} g_{j}=g_{k}$.

Therefore total number of extra vertices introduced to form $G_{2}(\Gamma)$ is equal to

$$
\sum_{i=1}^{n} \sum_{k=1}^{s}(2 k+3)=\sum_{i=1}^{n}\left(s^{2}+4 s\right)=n\left(s^{2}+4 s\right)
$$

Hence $\left|V\left(G_{2}(\Gamma)\right)\right|=n\left(s^{2}+4 s\right)+n=n\left(s^{2}+4 s+1\right)$.

Theorem 6. $G_{2}(\Gamma)$ has $n\left(s^{2}+5 s\right)$ edges, where $|\Lambda|=s$.

Proof. The number of edges in $G_{2}(\Gamma)$ is given by

$$
m=\sum_{i=1}^{n} \sum_{k=1}^{s}(k+2+k+2)=\sum_{i=1}^{n}\left(s^{2}+5 s\right)=n\left(s^{2}+5 s\right) .
$$

Theorem 7. $G_{2}(\Gamma)$ is not hyperenergetic.

Proof. We see that

$$
m=n\left(s^{2}+5 s\right)<2\left[n\left(s^{2}+4 s+1\right)\right]-2=2 p-2 .
$$

Thus the result follows from Theorem 1 .

Combining the above observations, we conclude with the following result.

Theorem 8. Every group is isomorphic to the automorphism group of some non-hyperenergetic graph.

\section{REFERENCES}

[1] I. Gutman, "The energy of a graph," Journal of the Serbian Chemical Society, Vol. 64, pp. 199-205, 1999.

[2] L. Lovasz, "Combinatorial problems and exercises," North-Holland, Amsterdam, 1979.

[3] I. Gutman and L. Pavlovic, "The energy of some graphs with large number of edges," Bull. Acad. Serbe Sci. Arts, Vol. 118, pp. 35-50, 1999

[4] I. Gutman Y. Hou, H. B. Walikar, H. S. Ramane, and P. R. Hamphiholi, "No huckel graph is hyperenergetic," Journal of the Serbian Chemical Society, Vol. 65, No. 11, pp 799-801, 2000.

[5] I. Gutman, "The energy of a graph, old and new results," In: A. Betten, et al., Algebraic Combinatorics and its Applications, Springer-Verlag, Berlin, pp.196-211, 2001.

[6] I. Gutman and L. Pavlovic, "The energy of some graphs with large number of edges," Bull. Acad. Serbe Sci. Arts, Vol. 118, pp. 35-50, 1999.

[7] R. Frucht, "Herstellung von graphin mit vorgege bener abstakten Gruppe," Compositio Mathematica, Vol. 6, pp. 239-250, 1938.

[8] R. Frucht, "Graphs of degree three with a given abstract group," Canadian Journal Mathematics, Vol. 1, pp. 365-378, 1949.

[9] R. Frucht and F. Harary, "On the corona of two graphs," Aequationes mathematicae, Basel, Vol. 4, pp. 322-325, 1970.

[10] J. Koolen, V. Moulton, I. Gutman, and D. Vidovic, "More hyperenergetic molecular graphs," Journal of the Serbian Chemical Society, Vol. 65, pp. 571-575, 2000.

[11] H. B. Walikar, H. S. Ramane, and P. R. Hamphiholi, "On the energy of a graph," Proceedings of Conference on Graph Connections (R. Balakrishnan et al. eds.), Allied Publishers, New Delhi, pp. 120-123, 1999. 